\title{
EIGEN BASED METHODS TO JOINTLY ESTIMATE FREQUENCY AND TIMING IN PSK AND MSK SIGNALS
}

\author{
M. Cabrera and M. A. Lagunas. \\ Dept. of Signal Theory and Communications, E.T.S.I. Telecomunicacion, UPC
} Apdo. 30002, 08080-Barcelona, SPAIN.

\begin{abstract}
Joint detection of carrier and timing parameters in digital communications is an alternative of recent interest when compared with individual acquisitions of each one of the parameters. In this paper the eigen value decomposition method of the signal structured covariance matrix is applied to jointly synchronize carrier frequency and timing (symbol transitions) in a full digital receiver. A very fast acquisition stage is obtained when PSK and MSK signals are transmitted in an additive gaussian noise channel. The parameters of interest are estimated from the signal subspace as a fast and computationally efficient alternative to the matched filter technique to estimate the timing parameter and also and alternative to the classic bank of filters technique used to acquire the carrier frequency parameter. $\left.{ }^{*}\right)$
\end{abstract}

\section{INTRODUCTION}

Digital Signal Processing Techniques are of growing interest in the field of Satellite Communications. A lot of digital methods have appeared to estimate the timing parameter in digital communications and from a Maximum Likelihood point of view, [1]. Of particular interest are those methods that obtain a joint timing and carrier phase estimation with single algorithm. Nevertheless, these algorithms are usually performed in a closed loop technique and in a digital environment neither a lot of efforts have been done to include carrier frequency acquisition in this stage nor the frequency is detected applying directly a Maximum Likelihood method in digital communication systems.

In order to accomplish a fast transmission of information, the estimation of the carrier parameters, frequency and phase, and also symbol transitions must be acquired as fast as possible, preferably by means of an open loop method and from a very short burst of symbols. Simultaneous Doppler frequency and symbol transition acquisition methods using very short data records, are proposed from an ML-estimation point of view.

The log-likelihood function is analyzed for a burst of symbols modulating a sinusoidal carrier in Phase Shif Keyed (PSK) or in Minimum-Phase Shift Keyed (MSK), in additive gaussian noise. The signal is sampled, to get a full digital structure for the processor. The vector obtained, when no data information is used in the estimation stage suits a multivariate normal model [2]. The resulting log-likelihood

${ }^{(*)}$ This work has been supported by PLANICYDT/TIC 302/89 function is just a quadratic form of the data vector and the covariance matrix. The parameters to be estimated are Doppler frequency $\omega_{\mathrm{D}}$ and symbol transition $\tau$ (also called timing), variables that parameterize the matrix covariance structure. In section 2 the complete log-likelihood function is analyzed, concentrating the of the interest in the covariance matrix of the signal.

From a burst of a received signal, different submatrices appearing in the complete covariance matrix are identified. Using a Singular Value Decomposition of these matrices, the symbol transition parameter is obtained from the eigenvalues that determine the signal subspace, while the corresponding eigenvectors steer the Doppler frequency. In section 3 this analysis is accomplished and also simulations results are presented. The resulting method is proposed as a concluding alternative to closed loop techniques. Finally the most important conclusions are presented in section 4

\section{LOG LIKELIHOOD FUNCTION}

The received signal has been processed before the synchronization stage. This means that it has been bandpass filtered, down translated to a I-Q base-band signal and analog to digital converted. The signal to be processed, $x(n)$, for the parameters identification is shown in (1) as a sequence of samples coming from a PSK burst of $\mathbf{N}_{\mathrm{sy}}$ symbols, sampled at $\mathrm{N}_{\mathrm{ss}}$ samples/symbol rate. The base-band pulse form has been initially assumed to be of limited duration, equal to one symbol period, in order to simplify the problem.

$x(n)=A \sum_{k=1}^{N_{s y}} e^{j\left(\phi_{k}+\phi_{c}\right)} p\left(n-k N_{s s}-\tau\right) e^{j \omega_{D} n}+n(n)=s(n)+n(n)$

The time variable has been normaliced to the sampling period. $s(n)$ is the desired signal sequence. It is parametericed by some unknown parameters, as the modulating phase $\phi_{k}$, the carrier phase $\phi_{c}$, the timing or symbol transition parameter $\tau$, and the Doppler frequency $\omega_{\mathrm{D}}$. The magnitude $A$ is not of great interest when constant modul signals are processed. In the following it will be considered equal to one. $n(n)$ is the noise signal, assumed as a complex gaussian distribution with independent real and imaginary parts of zero mean and $\sigma^{2} v$ ariance.

When $N_{s y}$ symbols are processed, a vector $X$ of $\mathrm{N}=\mathbf{N}_{s y} \times \mathrm{N}_{s s}$ order is obtained and it is distributed as $\mathrm{N}\left(\mathbf{S}, 2 \sigma^{2} \mathrm{I}\right)$ with desired signal vector $\underline{S}$ parametericed by the unknown parameters. 


$$
\underline{\mathrm{S}}=\underline{\mathrm{S}}\left(\omega_{\mathrm{D}}, \tau, \phi_{\mathrm{c}}, \mathcal{Q}\right) \quad \Phi=\left\{\phi_{\mathrm{k}} ; \mathrm{k}=1 . . \mathrm{N}_{\mathrm{sy}}\right\}
$$

To eliminate the phase components of the desired signal vector $\underline{S}$, the received signal $\underline{X}$ is averaged for all the equiprobable values of the transmitted symbols. The resultant log-likelihood function is thus simplified to a quadratic form as it is shown in section 2.1. for PSK modulation.

\subsection{Covariance matrices in PSK signals}

The phase in a PSK signal is modulated by a sequence of independent symbols. Assuming the equiprobability of the transmitted symbols proceeding of a limited alphabet, the mean averaged signal vector results equal to zero.

$$
\mathrm{E}\left[\mathrm{e}^{\left.\mathrm{j} \phi_{\mathbf{k}}\right]}=0, \mathrm{k}=1 . . \mathrm{N}_{\mathrm{sy}} ; \mathrm{E}[\underline{\mathrm{X}}]=\underline{0}\right.
$$

$E$ is the statistical operator. With (3) the parameterizacion of the signal by the phase parameters disappears. When the covariance matrix of the signal vector is calculated, the same kind of simplifications are obtained. Two samples $s(i)$ and $s(k)$ of one symbol are strongly correlated, but two samples proceeding of different symbols are completely uncorrelated because of the statistical independence between different symbols of the transmitted sequence.

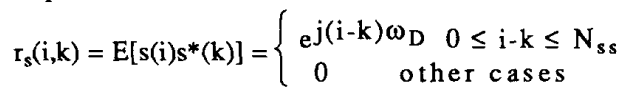

So, the non zero elements are distributed in the total covariance matrix along the principal diagonal, forming $\mathrm{N}_{\mathrm{ss}} \mathrm{XN}_{\mathrm{Ss}}$ submatrices, denominated $\underline{\mathrm{R}}_{\mathrm{o}}$. For a five symbols burst the general covariance matrix structure is shown in (5).

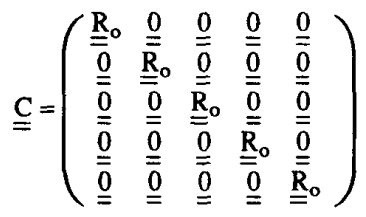

The submatrix $\underline{R}_{0}$ represents the autocovariance of a single symbol vector " $\mathrm{i} "$ and it is generated by the steering vector $\underline{V}\left(\omega_{D}\right)$ to the carrier Doppler frequency parameter $\omega_{D}$, caused by the constant envelope conditions of the PSK signal and by the noise components.

$$
E\left[\underline{X}(i) \underline{X}(i)^{H}\right]=\underline{R}_{o}=\underline{V}\left(\omega_{D}\right) \underline{V}^{H}\left(\omega_{D}\right)+2 \sigma^{2} \underline{I}
$$

$\mathrm{H}$ denotes the hermitian vector. Samples of two different symbols are uncorrelated. The timing parameter information is located in the special structure of the covariance matrix, which has a particular form considering null and non null elements. The elements of the principal diagonal appear periodically with period equal to the number of samples by symbol $\mathrm{N}_{\mathrm{ss}}$, and the same happens with the other diagonals parallel to the principal one. This characteristic is caused by the ciclostacionarity condition of this kind of digital modulation. The covariance matrix structure $\underline{\underline{C}}$ is thus parametericed by the Doppler frequency $\omega_{\mathrm{D}}$ steering the $\underline{R}_{0}$ submatrix and by the timing parameter $\tau$.
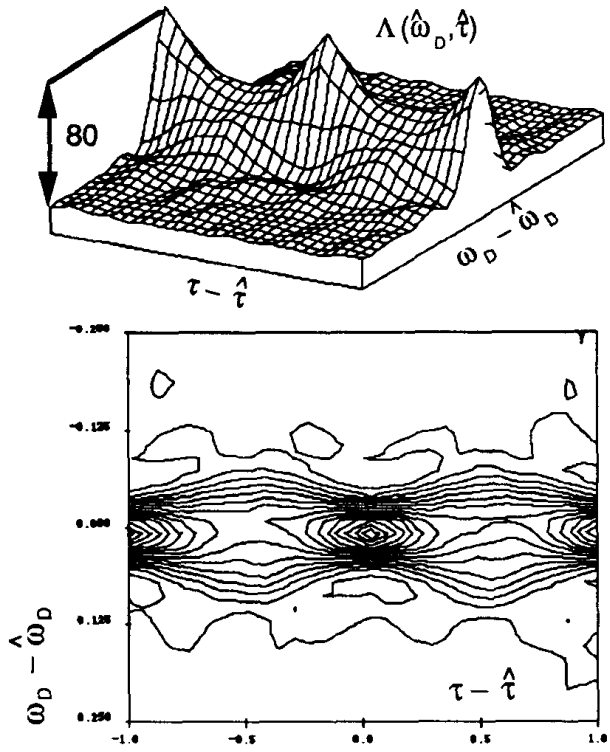

Fig. 1. ML function (nepers) with $\mathrm{SNR}=5 \mathrm{~dB}$.

The ML function evaluation, for a ten symbol burst of a PSK received signal with $5 \mathrm{~dB}$ of Signal to Noise ratio, is shown in figure 1 . One axe shows the Doppler frequency error, and the other the timing error. The timing error range is of two symbols, and the Doppler frequency range, when normalized to the sampling frequency, is 0,5 . It can be noticed that in the three correct synchronism situations (Doppler frequency error equal to zero and timing error equal to $-1,0,+1$ symbol) a maximum peak is located.

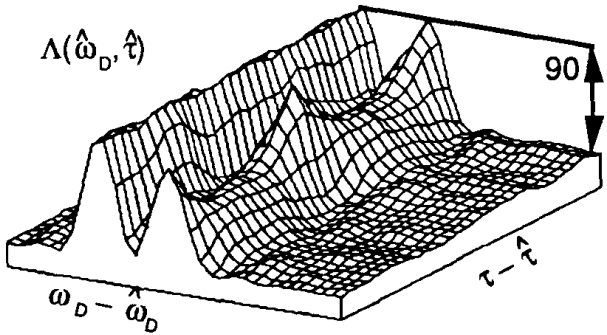

Fig. 2. $M L$ function (nepers) with $S N R=5 \mathrm{~dB}$, interference to signal ratio $0 \mathrm{~dB}$, interference frequency $\omega_{\mathrm{T}}=\omega_{\mathrm{D}}+0.1$.

When a sinusoidal interference is added to the desired signal, the log-ML function discriminates both signals, the PSK modulated carrier and the unmodulated carrier. As an example it is presented in figure 2, the case of an interfering frequency of 0,1 . Both signals are detected with complete resolution. 


\subsection{Covariance matrices in MSK signals}

For the MSK case, each pair of consecutive symbols in the sequence are dependent, so auto-covariance symbol submatrices and cross-covariance submatrices of each pair of consecutive symbols appear. The resultant structure is shown in (7) for a five symbol burst of an MSK signal. The non zero correlation of samples coming from two consecutive symbols is given by the codification produced in the instantaneous phase of the MSK signal, necessary to obtain a phase continuity.

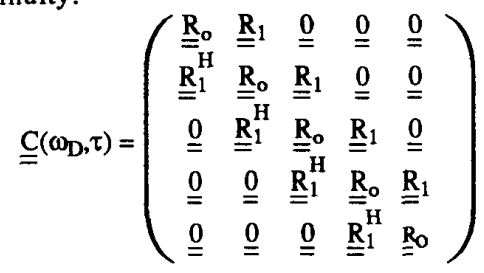

A periodical structure for all the diagonals in the matrix is obtained again. The signal vectors that generate the autocorrelation matrix $\underline{R}_{o}$ and the vector generator of the cross-correlation matrix $\underline{R}_{1}$, steer the positive modulation frequency $(\mathrm{V}(+))$ or the negative modulation frequency $(\mathrm{V}(-))$ added to the Doppler frequency $\omega_{\mathrm{D}}$.

$$
\begin{aligned}
& \underline{\underline{R}}_{o}=E\left[\underline{X}(i) \underline{X}(i)^{H}\right]=\frac{1}{2}\left(\underline{V}(+) \underline{V}^{H}(+)+\underline{\mathrm{V}}(-) \underline{V}^{H}(-)\right)+2 \sigma^{2} \underline{I} \\
& \underline{\underline{R}}_{1}=\mathrm{E}\left[\mathbf{X}(\mathrm{i}) \mathrm{X}(\mathrm{i}+1)^{\mathrm{H}}\right]=\frac{1}{4}(-\mathrm{j} \underline{\mathrm{V}}(+)+\mathrm{j} \underline{\mathrm{Y}}(-))(\underline{\mathrm{V}}(+)+\underline{\mathrm{V}}(-))^{\mathrm{H}} \\
& \underline{\mathrm{V}}( \pm)=\left\{\exp \left(\mathrm{ji}\left(\omega_{\mathrm{D}} \pm \frac{\pi}{2 \mathrm{~N}_{s s}}\right) ; \mathrm{i}=0, ., \mathrm{N}_{\mathrm{ss}}-1\right\}\right.
\end{aligned}
$$

In (10) both modulation frequencies are defined, the positive one and the negative one, for the MSK modulation [3]. The statistical dependence between consecutive symbols of MSK modulation has produced cross-covariance submatrices $R_{1}$ of two consecutive symbols, beside the diagonal of autocovariance symbol submatrices $\underline{\underline{R}}_{\mathbf{o}}$. Similar simulation results are obtained for MSK modulation compared with those presented for the PSK case.

\section{EIGEN VALUE DECOMPOSITION}

The complete evaluation of the log-likelihood function gives us all the information about both parameters of interest, frequency and timing. Nevertheless the computational cost to detect them directly from the maximum of the likelihood function is very high. An intensive search could not be accomplished in real time in a digital synchronizer.

In the evaluation of the likelihood function, implicitly we compare the theoretical submatrices of correlation $\underline{R}_{i}$ with the real submatrices of the processed burst. From a burst of a received signal, we propose to identify the different submatrices appearing in the complete covariance matrix, as an alternative to the full analysis of the likelihood function. With a Singular Value Decomposition of the obtained correlation submatrices, the symbol transition parameter and the Doppler frequency parameter are detected from the signal subspace.

\subsection{Signal Subspace in PSK}

The $\mathrm{N}_{\mathrm{sy}} \times \mathrm{N}_{\mathrm{ss}}$ order vector is divided in $\mathrm{N}_{\mathrm{sy}}$ vectors of $\mathrm{N}_{\mathrm{ss}}$ order. These vectors are averaged to get the matrix estimation $\underline{\underline{R}}_{0}$ in PSK signals.

$$
\underline{\underline{R}}_{o}=\frac{1}{N_{s y}} \sum_{i=1}^{N_{s y}} \underline{X}(i) \underline{X}(i)^{H}
$$

Different forms of averaging the vectors are possible, depending on the initial sample to be processed, this is, depending on the $t$ estimation assumed to compute $\underline{\underline{R}}_{0}$. When the burst of symbols is processed without timing error $\tau=t$ the estimation $\underline{\underline{R}}_{0}$ is only generated by the steering vector $\underline{V}\left(\omega_{D}\right)$ and the noise components, just as it is shown in (6). The signal subspace range is minimum and equal to one, and the signal vector is the eigenvector that generates the signal subspace. The associated eigenvalue is the eigenvector energy added to the noise power. The other $N_{s y}-1$ eigenvectors are noise components and they generate the called orthogonal subspace.

$$
\underline{\mathrm{e}}=\underline{\mathrm{V}}\left(\omega_{\mathrm{D}}\right) \quad \lambda=\underline{\mathrm{V}}^{\mathrm{H}}\left(\omega_{\mathrm{D}}\right) \underline{\mathrm{V}}\left(\omega_{\mathrm{D}}\right)+2 \sigma^{2}=\mathrm{N}_{\mathrm{ms}}+2 \sigma^{2}
$$

In non correct time synchronism situations, samples coming from two different symbols are uncorrelated and zero elements appear in the estimated submatrix. In (13), (14) an example is given with $\mathbf{N}_{\mathrm{ss}}=5$ samples/symbol and an initial timing error of two samples. The maximum eigenvalue determines the timing error and all the signal eigenvectors steer the Doppler frequency $\omega_{\mathrm{D}}$.

$$
\begin{aligned}
& \underline{e}_{1}^{H}=\left(1, e^{-j \omega_{D}}, e^{\left.-2 j \omega_{D}, 0,0\right)}, \quad \lambda_{1}=N_{m s}-(\tau-\hat{\tau})+2 \sigma^{2}\right. \\
& \underline{\mathbf{e}}_{2}^{\mathrm{H}}=\left(0,0,1, \mathrm{e}^{-j \omega_{\mathrm{D}}}\right), \lambda_{2}=(\tau-\hat{\tau})+2 \sigma^{2}
\end{aligned}
$$

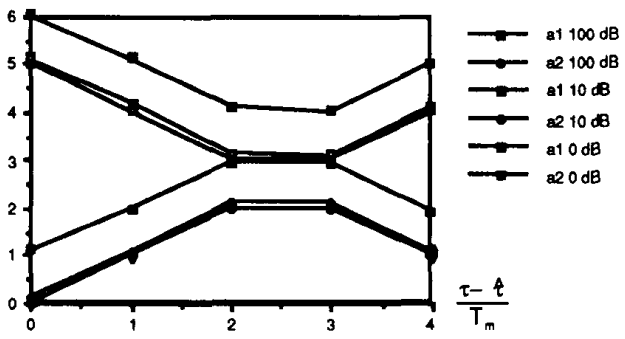

Fig. 3. Signal eigenvalues evolution with timing error. $\lambda_{1}=\mathrm{a} 1, \lambda_{2}=\mathrm{a} 2, \mathrm{~N}_{\mathrm{ss}}=5, \mathrm{~T}_{\mathrm{m}}$ sampling period.

The best eigenvectors to get the frequency value by spectral estimation techniques, are those obtained from the analysis of the covariance submatrix in a time synchronized situation. In figure 3 both signal eigenvalues evolution with timing error is shown for a burst of $\mathrm{N}_{\mathrm{sy}}=500$ symbols sampled at rate of $\mathrm{N}_{\mathrm{ss}}=5$ samples/symbol, with different signal to 
noise ratio values. The maximum eigenvalue $\lambda_{1}$ decreases with the timing error, while the second signal eigenvalue $\lambda_{2}$ appear and increases with the timing error.

The evolution of the signal eigenvalues with the number of symbols $N_{s y}$ used to compute the matrix has also been studied, and it has been noted that with a burst of 25 symbols and $S N R=0 \mathrm{~dB}$, the maximum eigenvalue is obtained with enough resolution and in consequence the timing parameter is correctly detected.

\subsection{Signal Subspace in MSK signals.}

If MSK signal is processed, there are two different submatrices available for computing the signal subspace the autocovariance matrix and the crosscovariance matrix. The estimated $\underline{\underline{R}}_{0}$ range in a correct timing synchronism situation is two and it increases to three in a non correct timing case. Signal eigenvalues and eigenvectors are given in [4]. The evolution of the two maximum signal eigenvalues with timing error is similar to the PSK case shown in precedent figures.

The range of the cross-covariance submatrix $\underline{R}_{1}$ in a correct timing assumption is equal to one and it increases to two when there is a timing error. In figure 4 the evolution of the corresponding signal eigenvalues is shown.

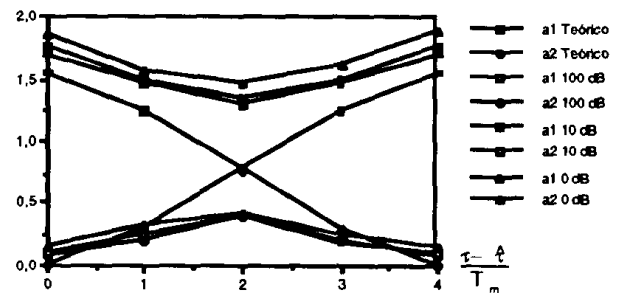

Fig. 4. $\underline{\underline{R}}_{1}$ signal eigenvalues evolution with timing error. $\lambda_{1}=\mathrm{a} 1, \lambda_{2}=\mathrm{a} 2, \mathrm{~N}_{\mathrm{ss}}=5, \mathrm{SNR}=0,10,100 \mathrm{~dB}$ and no noise.

It should be noted that the second signal eigenvalue $\lambda_{2}$ appears when timing error occurs. Theoretically in this matrix there is not noise subspace (AWGN channel), because it does not contain elements of the principal diagonal of the total covariance matrix $\underline{C}$, but in the simulation results presented in figure 4 , the computed eigenvalues in noisy situations are over the theoretical values obtained without noise. Nevertheless the evolution of the principal eigenvalue detects correctly the timing error in all the cases.

\subsection{Final proposed scheme}

To jointly obtain the frequency and the timing parameters directly from the signal subspace, the autocovariance symbol matrix $\underline{R}_{0}$ (or the cross-covariance symbol matrix $\underline{R}_{1}$ in MSK ) has to be computed from a symbol burst. By means of parallel processing techniques, assuming the $\mathrm{N}_{\mathrm{ss}}$ initial possible timing errors, the principal eigenvalue of each computed matrix is obtained. The maximum one detects the correct timing parameter and from the associated eigenvectors that generate the signal subspace the Doppler frequency is directly estimated. A first and row frequency estimation is obtained that could be improved in a second stage.

The fundamental advantage that this method presents in front of the classic matched filter can be summariced as follow. The signal eigenvectors represent the best matched filter to the received signal. The computed eigenvectors represent the impulse response that implicitly contains the possible transformations produced by the channel response and the maximum signal eigenvalue is the maximum and optimum correlation lag to detect the correct synchronism situation.

\section{CONCLUSIONS}

The introduction of the Doppler frequency in the maximum likelihood function of the processed PSK or MSK received signal can be considered an original application of the ML theory applied to the parameter acquisition stage. The sensibility of this function with noise and other undesired effects has been studied, proving that narrowband interference rejection and robustness with noise are very important characteristics of the detection system proposed.

The acquisition of the parameters from SVD techniques can be done using parallel processing to track the subspace signal eigenvalues as information for the timing parameter, and applying methods of spectral estimation based on SVD analysis (Pisarenko, MUSIC, etc..[5]) to acquire the Doppler frequency from the associated eigenvectors. The estimation of both parameters, frequency and timing directly from the subspace signal, gives to the problem a linear perspective, with consequent advantages in convergence and general resolution of the problem. They are proposed as an alternative replacing two isolated techniques for timing acquiring (matched filtering) and frequency estimation (bank of filters), with significant reduction of computation cost and with a very fast convergence from very short data records of signal.

Finally, let's say that a remarkable point is the easy extension of the method to more general modulation formats, those as CPFSK modulation in general and PSK signal with band limited transmission pulse (Nyquist Pulse Shaping). The extension of correlation between different symbols will be done in every case by codification of symbols (CPFSK) or by the extension in time of the transmission pulse (Nyquist pulse). From the resulting correlation the signal subspace must be determinate in any case.

\section{REFERENCES}

[1] F.M.Gardner. "Demodulator Reference Recovery Techniques Suited for Digital Implementation". European Space Agency. ESTEC Contract №6847/86/NL/DG, 1988.

[2] L. Sharf. "Statistical Signal Processing, Detection, Estimation, Time Series Analysis". Addison Wesley, 1991.

[3] A. Carlson. Communication Systems. McGraw Hill 1987.

[4] M. Cabrera. "Aplicación de Técnicas de DSP a Estimación de Parámetros de Sincronismo en Comunicaciones Digitales". Ph. D. Dissertation, TSC Dpt. UPC University, 1991.

[5] L. Marple "Digital Spectral Analysis" Prentice-Hall 1987. 